\title{
Iberoamérica y la Corte Penal Internacional: logros y retos de una interacción compleja e inacabada
}

\section{Latin america and the International Criminal Court: achievements and challenges of a complex and unfinished interaction}

\author{
Magdalena M. Martín ${ }^{1}$ \\ Universidad de Málaga (España)
}

Recibido: 25-03-14

Aprobado: 10-04-14

\section{Resumen}

Iberoamérica y la CPI mantienen desde hace más de dos décadas una interacción compleja y viva, con luces y sombras. Las expectativas que en esta región despiertan las actividades a desarrollar por la CPI a corto y medio plazo parecen indicar que mientras que África ha sido el continente estrella en la primera década de vida de la CPI, Iberoamérica puede ocupar un papel relevante cuando la CPI alcance su mayoría de edad. De ahí la idoneidad y oportunidad de nuestra contribución, en la que, al objeto de verificar esta hipótesis, repasaremos primero la aportación iberoamericana a la CPI, desde la ratificación e implementación del ER

\footnotetext{
${ }^{1}$ (magdalena@uma.es) Magdalena M. Martín Martínez es Catedrática acreditada de Derecho Internacional Público y Relaciones Internacionales en la Universidad de Málaga. Profesora Titular de Derecho Internacional Público, Derecho Comunitario y Relaciones Internacionales en la Universidad de Málaga desde 1997 y Doctora en Derecho por el Instituto Universitario Europeo de Florencia. Ha impartido conferencias y seminarios en las principales Universidades de España (Madrid, Barcelona, Sevilla, Salamanca, Santiago de Compostela etc.) y en Iberoamérica (Costa Rica, Perú, Venezuela). En la actualidad sus principales líneas de investigación tienen que ver con el Espacio Europeo de Libertad, Seguridad y Justicia y la acción exterior de la Unión Europea, la inmigración y el género, y el Derecho Internacional Penal. Entre sus publicaciones más recientes destacan: "Los crímenes de naturaleza sexual en el Derecho Internacional Humanitario", (co-autora Isabel Lirola), Instituto Catalán Internacional para la Paz Informes 3/2013, ISSN: 2013-9446, 106 pp; "Protección a las víctimas, violencia de género y cooperación judicial penal en la Unión Europea post-Lisboa", Revista de Derecho Comunitario Europeo, ISSN 1138-4026, Año no 15, No 39, 2011, pp. 407-442; "El principio de jurisdicción universal en el ordenamiento jurídico español: pasado, presente y futuro", Anuario Mexicano de Derecho Internacional, vol. XI, 2011, pp. 251-303 (co-autora Carmen Márquez Carrasco).
} 
hasta la Conferencia de Revisión de Kampala de 2010, para a continuación abordar la otra dimensión de esta interacción recíproca, analizando cuales han sido las principales aportaciones que el ER ha supuesto para la región, así como los retos y desafíos pendientes, finalizando nuestro examen con algunas reflexiones generales.

Palabras-clave: Iberoamérica, Corte Penal Internacional, Conferencia de Revisión de Kampala

\begin{abstract}
Latin America and the ICC held for more than two decades a complex and lively interaction full of lights and shadows. The current activities develop by the ICC suggest that, if African continent has monopolized the first decade of the CPI, Latin America can play a significant role in the short and medium term. For that very reason, this article focuses on the Latin American contribution to the ICC, from the negotiation of the Rome Statute till its entry into force and the process of implementation in domestic laws. Then this article analyses the other side of the coin, the contribution of the Rome Statute on the region, as well as the remaining challenges, completing our review with a final reflection.
\end{abstract}

Key-words: Latin America, International Criminal Court, Review Conference in Kampala.

\title{
Introducción
}

La conmemoración de cada nuevo aniversario de la entrada en vigor del Estatuto de Roma (ER) de la Corte Penal Internacional (CPI), acaecida el 1 de julio de 2002, ofrece un motivo adicional para aproximarnos a la labor que viene desempeñando esta Institución. De hecho, con ocasión del décimo aniversario celebrado en 2012 se produjo un significativo repunte en el número de aportaciones doctrinales al respecto, la mayoría de las cuales contenían balances dispares de los éxitos y fracasos cosechados por la $\mathrm{CPI}^{2}$. En una parte significativa de estos estudios, la metodología empleada partía de

\footnotetext{
${ }^{2}$ Véase sin ánimo de exhaustividad en español Amparo Martínez Guerra, Corte Penal Internacional: balance de una década, "Política Exterior" $\mathrm{n}^{\circ}$ 148, julio/agosto (2012), p.. 22-27; Federación Internacional de Derechos Humanos, Corte Penal Internacional 2002-2012: 10 años, 10 recomendaciones para una Corte Penal Internacional eficaz e independiente, disponible enhttp:// www.fidh.org/IMG/pdf/cpil0ansespagn.pdf; Claudia Cárdenas / Karinna Fernández, La Corte Penal Internacional y sus primeros 10 años: un enfoque práctico, Thomson Reuters. 2013; Carmen Quesada (coord.), Décimo Aniversario de la Corte Penal Internacional "Tiempo de Paz”, (2012). En lengua inglesa, Joseph Isanga, The International Criminal Court ten years later: appraisal and perspective, "Cardozo Journal of International and Comparative Law", 21 (2013), p. 235-323; Mireille Delmas-Marty, Ambiguities and Lacunae.The International Criminal Law ten years on, "Journal of International Criminal Justice", 11 (2013), p.553-561.
} 
la diferenciación del impacto de la CPI en los diferentes continentes o ámbitos geográficos regionales, haciendo especial hincapié en dos de ellos, África e Iberoamérica ${ }^{3}$.

La atención preferencial que el continente africano ha suscitado en los últimos tiempos tiene una explicación obvia, puesto que desde su creación hasta la actualidad la CPI ha abierto investigaciones en ocho situaciones, todas en el África subsahariana. Por orden cronológico: Uganda (2003); República Democrática del Congo (2004); República Centroafricana (2005); Darfur-Sudán (2005); Kenia (2010); Costa de Marfil (2011); Libia (2011) y Malí (2013). Esta unidireccionalidad y el sesgo exclusivo que supone han sido muy criticados, hasta el extremo de que la Unión Africana ha llegado a afirmar que se trata de una manifestación inequívoca de colonialismo judicial, o más gráficamente aún, una suerte de "caza racial"4.

El debate recurrente sobre la universalidad o no de la CPI nos retrotrae a la adopción del Estatuto de Roma y excede de los límites del presente trabajo, pese a lo cual consideramos necesario apuntar varios contraargumentos. Primero, que seis de las citadas situaciones han sido remitidas de forma voluntaria por los gobiernos de los Estados afectados (República Centroafricana, Costa de

\footnotetext{
${ }^{3}$ Frente a otras expresiones de uso común como Latinoamérica o Hispanoamérica, nos hemos decantado por Iberoamérica, que será la que empleemos en este artículo. La elección de una u otra no es neutral, por cuanto, además de la dimensión puramente lingüística, suele comportar otras consideraciones de índole política y geoestratégica. Por ejemplo, América Latina o Latinoamérica es un término francés del siglo XIX empleado para contrarrestar el termino y la influencia española de Hispanoamérica con el pretexto de dar cabida a todos los países en los que se hablaban lenguas romances, incluyendo el francés. Hispanoamérica se suele usar para designar a los países americanos en los que se habla español como lengua oficial o co-oficial, mientras que Iberoamérica sería en principio el término más adecuado, pues no tiene en cuenta a los países francófonos (Islas antillanas Haití, Guadalupe y Martinica) pero sí que incluye a los que, como Brasil, emplean el portugués. http:// castellanoactual.com/hispanoamerica-iberoamerica-y-latinoamerica/.

${ }^{4}$ Las discrepancias de la Unión Africana, con la CPI se remontan a 2009, con ocasión del procesamiento del presidente en activo sudanés Al-Bachir, pero se han recrudecido recientemente tras el procesamiento del también presidente en ejercicio de Kenia, Uhuru Kenyatta, y la apertura de juicio oral contra el vicepresidente William Ruto. De hecho, en la cumbre extraordinaria de esta organización que tuvo lugar en Addis Abeba los días 11 y 12 de octubre de 2013 Kenia propuso una denuncia masiva del Estatuto de Roma por parte de los miembros de la UA para forzar la suspensión de dichos procesamientos, así como la posibilidad de redefinir la competencia de la Corte Africana de Derechos Humanos al objeto de juzgar los crímenes internacionales de mayor gravedad. La CPI ha expresado su preocupación por esta situación, si bien ha tratado de diferenciar entre la posición de los gobiernos africanos y la de la sociedad civil, representada por las más de 130 ONGs que, con ocasión de esta polémica, reiteraron su apoyo a la CPI. Sobre esta cuestión cabe la consulta de Alexis Arieff, International Criminal Court Cases in Africa: Status and Policy Issues. Congressional Research Service, (2009), pp. 22 y ss; Rachel Goodman/ Nokukhanya Mncwabe, International Criminal Justice in Africa: Neocolonial agenda or strengthened accountability?, "African Transitional Justice Research Network", october (2011); Estephen Lamony, Is he International Criminal Court really picking on Africa?, http://africanarguments.org/2013/04/16/is-the-international-criminal-court-reallypicking-on-africa-by-stephen-a-lamony/; Francesca Benvenuto, Revuelta de los países africanos: El Tribunal Penal Internacional acusado, "Le Monde Diplomatique en español", nº 217, octubre (2013); Kai Ambos, ¿Corte Penal Internacional o Corte Africana?, Diario ABC, 28 de octubre de 2013.
} 
Marfil, República Democrática del Congo, Kenia, Mali y Uganda)5. Segundo, que existe un alto grado de presencia e influencia de África en la CPI, como demuestra el hecho de que 34 de los 54 miembros de la Unión Africana son Estados partes, y que la Fiscal jefe, F. Bansouda, y 4 de los 21 jueces actuales sean africanos ${ }^{6}$. Tercero, que un elevado aunque difícilmente cuantificable porcentaje de las víctimas de los crímenes objeto de la competencia de la Corte se localizan en este continente. Y por último, pero no menos importante, que la CPI pivota sobre el principio de complementariedad, actuando solo cuando, como sucede en las situaciones investigadas en África, se ha verificado que las jurisdicciones nacionales no quieren o no pueden conocer de dichos crímenes.

Sin ser tan notorios, los nexos entre la CPI e Iberoamérica han despertado también un considerable interés tanto en el plano académico como en el político ${ }^{7}$, no en vano dos de los exámenes preliminares abiertos por la Fiscalía, y todavía inconclusos, afectan a Colombia y Honduras ${ }^{8}$. Las expectativas que en esta región despiertan las actividades a desarrollar por la CPI a corto y medio plazo parecen indicar que si África ha sido el continente estrella en la primera década de vida de la CPI, Iberoamérica puede ocupar un papel relevante cuando la CPI alcance su mayoría de edad. Además de un pasado reciente en el que los regímenes dictatoriales y los conflictos armados fueron terreno abonado para la comisión de crímenes internacionales, tanto en África como en Iberoamérica existe hoy una necesidad compartida de poner fin a la impunidad, y una conciencia creciente entre la sociedad la sociedad civil y las victimas de que tienen que empoderarse para impulsar la labor de la justicia nacional e internacional. De ahí la idoneidad y oportunidad de nuestra contribución, en la que, al objeto de verificar esta hipótesis, repasaremos primero la aportación iberoamericana a la CPI, desde la ratificación e implementación del ER hasta la Conferencia de Revisión de Kampala de 2010, para a continuación abordar la otra dimensión de esta interacción recíproca, analizando cuales han sido las principales aportaciones que el ER ha supuesto para la región, así como los retos y desafíos pendientes, finalizando nuestro examen con algunas reflexiones generales.

\footnotetext{
${ }^{5}$ Por su parte la Fiscalía acordó motu proprio la remisión de las situaciones en Kenia y Costa de Marfil y el Consejo de Seguridad de la ONU el que reenvió a la CPI las situaciones de Darfur-Sudány Libia.

${ }^{6}$ La Fiscal Jefe Fatou Bensouda es de Gambia, y los cuatro jueces que a día de hoy componen las diferentes divisiones judiciales provenientes del África subsahariana son: Monageng (Bostwana, primer vice-presidente); Aluoch (Kenia); Eboe-Osuji (Nigeria); y Kuenyehia (Ghana).

7 José A. Guevara/ Narciso Dalmaso, La Corte Penal Internacional. Una visión iberoamericana, Porrúa-Universidad Iberoamericana, México, 2005; Nicolás Boeglin / Julia Hoffmann / Juan C. SáinzBurgo (ed.), La Corte Penal Internacional: una perspectiva latinoamericana, CPI-Universidad para la paz, Costa Rica, 2012.

${ }^{8}$ De conformidad con el Informe de la CPI sobre sus actividades en 2012-13 (A/68/314, de 13 de agosto de 2013), en este periodo la Fiscalía inició un examen preliminar de la situación de buques de matrícula de la Unión de las Comoras, la República Helénica y el Reino de Camboya; continuó los exámenes preliminares en el Afganistán, Colombia, Georgia, Guinea, Honduras, la República de Corea, así como en Nigeria, y concluyó su examen preliminar de la situación en Malí.
} 


\section{La aportación de Iberoamérica a la CPI: de Roma a Kampala}

En las investigaciones llevadas a cabo en el campo de las Ciencias Sociales resulta determinante la distancia cronológica, el intervalo temporal que separa al observador de lo observado, razón por la cual suele ser habitual recurrir a la periodización, a la fijación de etapas, para ordenar y facilitar la valoración del fenómeno analizado. Es por ello que, en perspectiva histórica, cabe afirmar que la posición mantenida tanto por los gobiernos de los países iberoamericanos como por la sociedad civil, desde la Conferencia diplomática de plenipotenciarios celebrada en Roma entre el 15 de junio y el 17 de julio de 1998 hasta la primera Conferencia de revisión que tuvo lugar en Kampala (Uganda) del 31 de mayo al 11 de junio de $2010^{9}$, puede calificarse de activa y comprometida con la CPI.

En efecto, el continente americano ha estado a la vanguardia desde los "orígenes contemporáneos" de la negociación del ER, puesto que fue una coalición de 16 países de la región la que, en el marco de la Asamblea General de la ONU, solicitó a la Comisión de Derecho Internacional que al debatir el Proyecto de Código de Crímenes contra la Paz y la Seguridad de la Humanidad se ocupase también de la cuestión del establecimiento de un tribunal u otro mecanismo de justicia penal internacional ${ }^{10}$. En concreto, la iniciativa fue promovida por Trinidad y Tobago, que veía en la jurisdicción universal una respuesta plausible ante la manifiesta debilidad estatal para hacer frente al tráfico ilícito de estupefacientes a gran escala, y la multiplicación de las mafias que desafiaban la capacidad de las jurisdicciones nacionales ${ }^{11}$.

Una vez abierto el proceso de adopción del ER es también notorio que los países iberoamericanos formaron un grupo propio, el "Grupo Latinoamericano y del Caribe" (GRULAC), en sintonía con otro más amplio denominado de los estados afines o "like minded". La composición heterogénea del mismo

\footnotetext{
${ }^{9}$ El artículo 123 del propio Estatuto preveía que trascurridos los siete primeros años desde la entrada en vigor, podrían proponerse enmiendas al texto original, que deberían ser aprobadas en una Conferencia de revisión que de hecho convocó el Secretario General, a la que asistieron más de 2000 personas, delegaciones de 67 Estados Partes, además de diferentes observadores, entre los que destacaron las delegaciones de Palestina, de 17 Estados no Partes y de cientos de ONGs. Vid. Documento de Opinión del Instituto de Estudios Estratégicos 10/2010, La Conferencia de Revisión del Estatuto de Roma de la Corte Penal Internacional; José L. Rodríguez Villasante, Nota sobre la Conferencia de Revisión del Estatuto de Roma de la Corte Penal Internacional, Kampala, Uganda, http://www.cruzroja.es/dih/pdfs/temas/3 4/3 4.pdf.

${ }^{10}$ Resolución A/44/39, de 4 de diciembre de 1989 , Responsabilidad penal internacional de las personas y entidades que participan en el tráfico ilícito de estupefacientes y en otras actividades delictivas trasnacionales: creación de un tribunal penal internacional que tenga jurisdicción sobre esos delitos, http://daccess-dds-ny.un.org/doc/RESOLUTION/GEN/NR0/555/21/IMG/NR055521. pdf?OpenElement.

${ }^{11}$ Sobre el proceso de establecimiento de la CPI y en concreto sobre la recuperación del objetivo de una jurisdicción penal permanente Isabel Lirola / Magdalena Martín, La Corte Penal Internacional. Justicia versus Impunidad, Ariel, Barcelona, 2001, pp. 47 y ss.
} 
y el hecho de que sus integrantes no mantuvieran una uniformidad absoluta, sino una afinidad o consenso en torno a las cuestiones cruciales tales como los crímenes objeto de la competencia o las modalidades de ejercicio de la acción penal, permitió el mantenimiento de posiciones particulares como las sostenidas por Argentina o México.

La impronta del GRULAC se hizo notar tanto es aspectos sustantivos como procesales del texto del ER. Respecto a los primeros, destaca la aceptación de la improcedencia del cargo oficial y la renuncia a la inviolabilidad de los Jefes de Estado prevista en el artículo 27 del ER al que luego nos referiremos, especialmente relevante por las dificultades que su incorporación planteaba en sus ordenamientos internos. En el ámbito procesal, fue determinante la insistencia en la promoción del multilingüismo, reflejada con éxito en el artículo 50 y la Regla 41 de las Reglas de Procedimiento y Prueba, en los que se consagra el español como lengua oficial, junto a los demás idiomas de las Naciones Unidas, lo que a su vez comporta la exigencia de que los fallos y otras decisiones fundamentales se publiquen en nuestro idioma, sin perjuicio del dominio del inglés y el francés como idiomas de trabajo.

En consonancia con la posición mantenida durante la negociación, la mayoría de los países iberoamericanos votaron el 17 de julio de 1989 a favor de la adopción del ER (120 a favor, 7 en contra y 21 abstenciones), si bien destacó la abstención de México, que se justificó alegando no disponer del texto definitivo hasta 24 horas antes de la votación y haber manifestado previamente sus discrepancias con la no inclusión de las armas nucleares en la lista de armas prohibidas y la admisión del opting out en los crímenes de guerra ${ }^{12}$. El primer país iberoamericano en depositar el instrumento de ratificación, y el noveno en la lista de los 60 exigidos por el artículo 126 para la entrada en vigor del ER fue Venezuela (7 de junio del 2000), al que en menos de un año después siguieron Argentina, Paraguay y Costa Rica. En febrero de 2014, de los 17 Estados de la región 15 han ratificado el ER ${ }^{13}$, y solo dos, El Salvador y Nicaragua, aún no lo han hecho, si bien en ambos casos puede considerarse una cuestión de tiempo, como demuestra el que oficialmente la propia CPI manifieste de forma retórica que estos dos países "todavía no han finalizado el proceso de ratificación".

La ausencia de El Salvador es poco comprensible, pues llegó a presidir el GRULAC y votó a favor de la adopción del ER. Además, el precedente de la ratificación de Guatemala en abril de 2012,con una trayectoria histórica

\footnotetext{
${ }^{12}$ Sergio González Gálvez, México y la Corte Penal Internacional, "Revista Mexicana de Política Exterior”,nº55-56 (1999), pp. 44-67; Sergio García Ramírez, Cuestiones constitucionales a propósito de la Corte Penal Internacional, "Cuestiones Constitucionales", nº 6 (2002), pp. 175-189;

${ }^{13}$ Los quince países iberoamericanos que han ratificado el ER son, por orden alfabético, Argentina, Bolivia, Brasil, Chile, Colombia, Costa Rica, Ecuador, Guatemala, Honduras, México, Panamá, Paraguay, Perú, Uruguay y Venezuela. Sobre el particular, Nicolás Boeglín, A 10 años de la entrada en vigor del Estatuto de Roma: Breves reflexiones desde una perspectiva latinoamericana, "Revista Judicial de Costa Rica”, no 107 (2013), pp. 13-21.
} 
reciente muy similar, demuestra que el final del conflicto interno salvadoreño y el inicio de un complejo proceso de reconciliación nacional no supone un obstáculo insalvable, ya que en virtud de la irretroactividad sancionada en el artículo 24, la CPI es competente para conocer de los crímenes cometidos desde la entrada en vigor del ER ( 1 de julio de 2002), aunque sin duda en la inacción inicial de El Salvador tuvo un efecto negativo la firma en 2002 de un Acuerdo Bilateral de Inmunidad (BIA) con los Estados Unidos de América ${ }^{14}$. Sea como fuere, tras conocerse que el informe sobre la compatibilidad con el derecho interno, encargado por el gobierno en 2010 y finalizado en 2012, solo ha detectado impedimentos menores y subsanables, la ratificación es una decisión de voluntad política.

En lo que concierne a Nicaragua, la conclusión es idéntica, aunque el objetivo de la ratificación inmediata parece más lejano por varias razones. Entre otras, Nicaragua ni siquiera firmó el ER, concluyó tempranamente, en junio de 2003, un Acuerdo de Inmunidad (BIA) con los Estados Unidos, y a mayor abundamiento el partido sandinista, que lideró el cambio socio político y que actualmente está en el gobierno, se ha mostrado siempre reacio a apoyar la labor de la CPI, hasta el punto de admitir que la ratificación del ER no está incluida en su agenda política. A semejanza de El Salvador, la reticencia nicaragüense carece de fundamento jurídico, en la medida en que se vincula a la comisión de crímenes de guerra en la guerra civil que asoló el país en años ochenta del pasado siglo.

Junto a estos dos países hay un tercero, Cuba, que a efectos de división regional no está incluida en Iberoamérica sino en el Caribe, cuya ratificación se vislumbra más compleja. A su antagonismo irresuelto con los Estados Unidos, que se tradujo en la Conferencia de Roma en un rechazo frontal al papel atribuido en el ER al Consejo de Seguridad de la ONU, hay que sumar una nueva disconformidad con la definición restringida del crimen de agresión adoptada tras la Conferencia de Kampala ${ }^{15}$.

Una vez que el ER entró en vigor, el GRULAC mantuvo su activismo, coordinando posiciones para intentar que los sistemas y tradiciones jurídicas comunes de sus miembros quedasen incorporados en las Reglas de Procedimiento y Prueba y en los Elementos de los Crímenes, instrumentos esenciales para que la CPI pudiera ejercer su función con efectividad y eficacia. También trató de

\footnotetext{
${ }^{14}$ Nelson Vaquerano / Jaime Martínez, El Salvador: Informes Nacionales, en Kai Ambos y Ezequiel Malarino (ed), Persecución Penal Nacional de Crímenes Internacionales en América Latina y España, Fundación Konrad Adenauer, Uruguay, 2003, pp. 305-335; Belissa Guerrero Silva, El Estatuto de Roma y su constitucionalidad en el Salvador, "Cuadernos Electrónicos Derechos Humanos y Democracia", nº4 (2008), pp. 33-56.

${ }^{15}$ Así se desprende al menos del tenor de lo manifestado por su representante, la embajadora Zelmys María Domínguez Cortina, durante la Asamblea de los Estados Partes de noviembre de 2013, en la que Cuba ha seguido participando en su condición de observador, http://www.icc-cpi.int/iccdocs/ asp_docs/ASP12/GenDeba/ICC-ASP12-GenDeba-Cuba-SPA.pdf.
} 
asegurar la presencia de nacionales en la elección de los primeros funcionarios de la $\mathrm{CPI}^{16}$, tarea en la que tuvo éxito con la elección en marzo de 2003 de 3 juristas de Brasil, Bolivia y Costa Rica entre los 18 primeros jueces, y, sobre todo, con la designación en junio de 2003 del fiscal argentino Luis Moreno Ocampo como primer Fiscal Jefe de la CPI.

Esta unidad de acción durante el primer decenio de vida de la CPI se ha roto sin embargo justo con la Conferencia de Kampala, que marca el inicio de una segunda y decisiva fase, al aflorar tensiones internas dentro del GRULAC que amenazaron incluso con fracturar en dos el grupo, los países del Caribe por un lado y los iberoamericanos por otro. El efecto negativo inmediato ha sido la disminución de su capacidad de influencia y la ausencia de un consenso sólido como el alcanzado en el seno de la UE. A juicio de Escobar Hernández, los dos ejemplos más significativos de este disenso serían el fracaso de la llamada propuesta ABS (argentino-brasileñosuiza) sobre la activación en dos tiempos del artículo 121 en relación con la agresión ${ }^{17}$, que derivó en una suerte de guerra entre los dos líderes del continente para ver cuál controlaba el grupo, y las divergencias en el proceso de renovación de los jueces, que estuvo a punto de saldarse con la pérdida del puesto asignado a la región ${ }^{18}$.

Con todo, pese a ser preocupante, la constatación de que existen discrepancias crecientes puede entenderse como una circunstancia meramente coyuntural si tenemos en cuenta que el compromiso diplomático de los países iberoamericanos ha fructificado merced a venir acompañado de una implicación constante de la organización internacional más importante de la región, la Organización de los Estados Americanos (OEA), y del soporte que la CPI conserva en la sociedad civil, canalizada a través de la acción de una pléyade de $\mathrm{ONGs}^{19}$, así como en la doctrina internacionalista iberoamericana ${ }^{20}$.

\footnotetext{
${ }^{16}$ Silvia Fernández de Gurmendi, El aporte de Iberoamérica a la CPI, en Héctor Olásolo / Salvador Cuencia, Perspectiva Iberoamericana sobre la Justicia Penal Internacional, Tirant Lo Blanch, Valencia, 2012, pp. 15-18.

${ }^{17}$ Gloria Fernández Arribas, Entrada en vigor y ejercicio por la Corte Penal Internacional de la jurisdicción sobre el crimen de agresión, Revista Electrónica de Estudios Internacionales $\mathrm{n}^{\circ} 25$ (2013), pp. 11 y ss, http://www.reei.org/index.php/revista/num25/notas/entrada-vigor-ejercicio-cortepenal-internacional-jurisdiccion-sobre-crimen-agresion.

18 Concepción Escobar Hernández, El futuro de la Corte Penal Internacional. Hacia el X aniversario de la entrada en vigor del Estatuto de Roma, Relatoría del Seminario, Documento 9/2012, Instituto Catalán Internacional para la Paz, 2012, p. 22.

19 El protagonismo de la OEA no puede ocultar la acción de otras organizaciones regionales con objetivos específicos como el CARICOM, UNASUR o MERCOSUR ni por supuesto el decisivo rol de las ONGs, en especial en lo relativo a la capacitación de los operadores jurídicos de la región, la difusión de la labor de la CPI y la publicación de manuales sobre implementación y cooperación. En este sentido cabe reseñar que en el Anexo IV al Acta Final de la Conferencia de Roma (A/CONF.183/10), que contenía la lista de ONG presentes figuraban la Asociación Americana de Juristas; Asociación Interamericana de Servicios Jurídicos; Asociación Internacional de Juristas Latinoamericanos; Comité Latinoamericano y del Caribe para la Defensa de los Derechos de la Mujer (CLADEM); y, por supuesto la Sección Americana de la Coalición para la Corte Penal Internacional. Juli Minoves-Triquell, ONG y pequeños estados en el establecimiento y consolidación de la CPI, "Revista CIDOB d'Afers Internacionals", no 101 (2013) pp. 177-193.

20 Iberoamérica y la Corte Penal Internacional: debates, reflexiones y preguntas, Luis Martí
} 
A diferencia de la posición crítica e incluso desafiante de otras organizaciones regionales como la Unión Africana, la OEA se ha caracterizado por un apoyo político permanente a la CPI, como atestigua el acervo de resoluciones aprobadas periódicamente por su Asamblea General. Tras la entrada en vigor del ER dicho apoyo se intensificó en un paquete de resoluciones específicas llamadas genéricamente de "Promoción de la Corte Penal Internacional", de la cual es exponente la Resolución 2728 (XLII-O/12), de 4 de junio de 2012 21 . En ella la Asamblea General de la OEA, tras reiterar su llamamiento a favor de la universalidad dela CPI y la obligatoriedad de su implementación por los miembros de la OEA que son parte en el ER, da cuenta de dos avances sustanciales en materia de cooperación, a saber: la conclusión el 18 de abril de 2011 de un acuerdo marco de cooperación entre la CPI y la Secretaría General de la OEA, y la firma un año después, el 25 de abril de 2012 de otro acuerdo de cooperación entre la CPI y la Comisión Interamericana de Derechos Humanos ${ }^{22}$.

Lejos de considerarse un objetivo cumplido, la necesidad de fortalecer la cooperación activa con la CPI sigue siendo una tarea pendiente en la OEA, como demuestra la reunión tripartita mantenida en Washington el 12 de abril de 2013 entre el Presidente de la CPI, la presidenta de la Asamblea de los Estados Partes y la Comisión de Asuntos Jurídicos y Políticos de la OEA, que desde 2002 viene celebrando sin interrupción sesiones anuales de trabajo sobre la CPI. En el transcurso de la misma se fijaron tres objetivos prioritarios con vistas a los próximos años. Por una parte, conseguir que los países iberoamericanos que son partes en el ER y que todavía no han ratificado el Acuerdo sobre los Privilegios e Inmunidades de la Corte Penal Internacional (APIC) lo hagan sin demora ${ }^{23}$. Por la otra, instar a la celebración de acuerdos bilaterales de cooperación sobre materias diversas relativas a la reubicación de testigos, la ejecución de sentencias o la aceptación de personas absueltas, en línea con el primer acuerdo de ejecución de sentencias en la región suscrito entre Colombia y la Corte Penal Internacional el 18 de mayo de 2011. Y en tercer lugar, que

Mingarro / Fernando Carrillo Flórez (ed.), Colección textos de jurisprudencia, Bogotá, 2006.

21 Entre el conjunto de las citadas Resoluciones destacan la Resolución AG/RES. 2072 (XXXVO/05). Promoción de la Corte Penal Internacional; la Resolución AG/RES. 2218 (XXXVI $\mathrm{O} / 06$ ). "Observaciones y recomendaciones al Informe Anual del Comité Jurídico Interamericano"; y la Resolución AG/RES. 2176 (XXXVI O/06). Promoción de la Corte Penal Internacional.

${ }^{22}$ El texto original del acuerdo se firmó en inglés y contempla la posibilidad de proveer a la otra parte, y en especial a la Fiscalía de la CPI, de información sobre decisiones, resoluciones, sentencias, informes y documentos, que pueda resultar útil para el procesamiento de casos y la ejecución de los mandatos de ambas instituciones, http://www.oas.org/es/cidh/prensa/comunicados/2012/039.asp. Mauricio Herdocia Sacasa, Los trabajos en el Sistema Interamericano: hacia una nueva Síntesis del DIH, La Corte Penal Internacional: Una perspectiva Latinoamericana, op. cit., pp. 82 y ss.

${ }^{23}$ A finales de 2012 los países iberoamericanos que habían ratificado el APIC eran Argentina, Bolivia, Brasil, Colombia, Costa Rica, Ecuador, Paraguay, Panamá, Perú, Uruguay y Venezuela. http://www.iccnow.org/documents/CICC_APIClist_current_sp_\%2872\%29.pdf. 
la OEA apoye económicamente al Fondo del Fideicomiso para las Víctimas e invite a sus miembros a ratificar también las enmiendas introducidas en Kampala sobre la definición del crimen de agresión y los crímenes de guerra en conflictos armados no internacionales ${ }^{24}$.

Junto a los desacuerdos surgidos en Kampala, el principal obstáculo para que la OEA consolide su política de cooperación con la CPI estriba en la oposición de los miembros de la organización que no son partes en el ER, en particular Cuba y los Estados Unidos de América, que han llegado a rechazar que el presupuesto ordinario de la organización se utilizara para financiar actividades de apoyo a la CPI. A ello se le añade la extraordinaria capacidad de presión diplomática, económica y militar ejercida por los Estados Unidos durante los primeros años de la administración Bush, que forzó a varios de sus socios en la OEA (en concreto Bolivia, Colombia, El Salvador, Honduras, Nicaragua, Panamá y República Dominicana) a la conclusión de acuerdos de inmunidad (BIAS) ${ }^{25}$. Aquellos otros que se resistieron a prestar dicha colaboración tuvieron que asumir costes elevados de diversa índole ${ }^{26}$.

El análisis del alcance y las consecuencias de la hostilidad estadounidense hacia la CPI, así como la evolución experimentada del contundente rechazo frontal en los orígenes hasta el tibio pero explícito reconocimiento actual de la labor de la CPI, exceden del presente trabajo ${ }^{27}$. No obstante, procede apuntar que el mandato del presidente Obama ha supuesto un giro ${ }^{28}$, que en el plano

${ }^{24}$ Comisión de Asuntos Jurídicos y Políticos. Sesión de trabajo sobre la Corte Penal Internacional. [AG/RES. 2728 (xlii-o/12)], viernes, 12 de abril de 2013; Consejo Permanente de la OEA/Ser.GCP Organización de los Estados Americanos CP/CAJP-3106/12 rev. 4 add. 5, 6 agosto 2013. También ICC-CPI-20130416PR897 Comunicado de prensa 16.04.2013 http://www.icc-i.int/en_menus/icc/ press\%20and\%20media/press\%20releases/Documents/pr897/130416-PRESS-RELEASE-ICC-andASP-President-address-OAS-Esp.pdf.

${ }_{25}$ Por el contrario, un número importante de miembros de la OEA (Argentina, Brasil, Costa Rica, Ecuador, México, Paraguay, Perú, Uruguay y Venezuela) rechazaron la firma de acuerdos similares. Al respecto, Carlos Viguria, Acuerdos bilaterales de inmunidad celebrados entre Estados Unidos y países miembros de la OEA,http://www.academia.edu/1826043/Acuerdos bilaterales de Inmunidad celebrados entre Estados Unidos y paises miembros de la Organizacion de_Estados_Americanos. El texto de los casi 100 BIAS concluidos por los Estados Unidos puede encontrarse en http://www.ll.georgetown.edu/guides/article 98.cfm y en: http://www.amicc.org/ usinfo/administration_policy_BIAs.html.

${ }^{26}$ La reciente publicación de los cables de Wikileaks ha sacado a la luz el alto precio pagado por los países objetores. Por ejemplo, en el caso de Costa Rica la negativa a firmar un acuerdo de inmunidad con los Estados Unidos supuso que entre 2004 y 2006 se le retirase la ayuda militar y quedara excluida del reparto de otros fondos económicos, perdiendo así varios millones de dólares hasta que a finales de 2006 la administración Bush advirtió los efectos contraproducentes de esta estrategia.

${ }^{27}$ David Scheffer, America's Embrace of the International Criminal Court, JURIST - Forum, July 2, 2012, http://jurist.org/forum/2012/06/dan-scheffer-us-icc.php; Francesca Varda, Working together for justice, La Corte Penal Internacional: una perspectiva Latinoamericana, op. cit., pp. 384-403.

${ }^{28}$ El giro de los Estados Unidos comenzó en los últimos años de la administración Bush pero se concretó durante la presidencia de Obama, primero con la derogación de los artículos de la ASPA que prohibían cooperar con aquellos países que se habían negado a firmar los acuerdos de inmunidad o acuerdos antídotos, y luego con la no inclusión de la llamada "Enmienda Nethercutt" en los Presupuestos. Prueba de implicación personal del presidente, su declaración de apoyo 
bilateral ha traído consigo el fin de las represalias contra aquellos países que no secundaron su boicot al ER, y que en el plano multilateral ha permitido a la OEA mantener un compromiso activo, aunque no pleno, de colaboración con la CPI. La renuncia de los Estados Unidos a seguir desempeñando el papel de enemigo público número uno de la $\mathrm{CPI}$ abre un escenario esperanzador pero no exento de interrogantes. Ya no es posible escudarse ni culpar a terceros, por lo que los países iberoamericanos devienen por vez primera plenamente responsables de la decisión de mantener su compromiso individual y multilateral en la lucha contra la impunidad en un momento especialmente delicado en el que la CPI, al encarar su segunda década de existencia, parece dispuesta a ampliar su punto de mira más allá de los confines del continente africano. Así pues, la pregunta decisiva es si Iberoamérica, además de reconocer la importancia que la puesta en marcha de la CPI ha supuesto para sus sistemas nacionales y regionales de justicia, está dispuesta a aceptar ser la destinataria del ejercicio de su jurisdicción.

\section{La aportación de la CPI en Iberoamérica: logros y desafíos}

El balance provisional de labor de la CPI en Iberoamérica nos obliga a confrontar las expectativas en su día suscitadas con los logros alcanzados, y, a su vez, a comparar éstos con los desafíos aún pendientes. En este sentido, desde la adopción del ER se observó que potencialmente la CPI estaba llamada a desempeñar un papel relevante en la región, por dos razones conexas. Primera, por el historial acumulado de violaciones graves y masivas de los derechos humanos, que indicaban la posible comisión de presuntos crímenes objeto de la competencia de la CPI. Y segunda, por la impunidad de la que gozaban muchos de los presuntos autores de dichos crímenes.

Respecto a la primera razón, conviene recordar que, como ya hemos avanzado, la irretroactividad impediría que la CPI pudiera haber conocido de las conductas constitutivas de genocidio, crímenes de lesa humanidad y crímenes de guerra perpetradas durante los regímenes dictatoriales que gobernaron la región antes de la entrada en vigor del $\mathrm{ER}^{29}$. No obstante, el mapa de conflictos

a la CPI a propósito del examen de la situación en Kenia, Statement by President Obama on the International Criminal Court announcement, december 15, 2010, http://www.whitehouse.gov/thepress-office/2010/12/15/statement-president-obama-international-criminal-court-announcement.

${ }^{29}$ En el Informe Anual 2013sobre el estado de los derechos humanos en el mundo de Amnistía Internacional se afirma que "las violaciones generalizadas de derechos humanos cometidas en el pasado, y la falta de rendición de cuentas por parte de los responsables, han arrojado una larga sombra sobre muchos países de la región. Sin embargo, en Argentina, Brasil, Guatemala y Uruguay tuvieron lugar procesamientos clave que señalaron importantes avances en la búsqueda de justicia por las violaciones cometidas durante los gobiernos militares pasado". http://www.es.amnesty.org/nuestrotrabajo/informe-anual/tendencias-regionales/america/. En este sentido cabe recordar la condena a 25 años de prisión al ex presidente peruano Alberto Fujimori, la condena a cadena perpetua al dictador 
armados del año 2012 contabiliza 38 "contextos bélicos", la mayoría en África (13) y Asia (12), seguidos por Oriente Medio (siete), Europa (cinco) y América (uno), localizado en Colombia. En concreto, el colombiano es calificado como un conflicto interno internacionalizado activo desde 1964, de intensidad decreciente y en proceso de solución ${ }^{30}$. El mismo mapa considera una situación, si no bélica, al menos de clara inestabilidad social, la padecida desde 2010 en Ciudad Juárez, México ${ }^{31}$, exponente máximo de la violencia sistemática y grave contra la mujer que ha llevado a la tipificación de estos homicidios por razón de género como delitos de feminicidio o femicidio en siete países iberoamericanos, así como en la jurisprudencia de la Corte Interamericana de Derechos Humanos ${ }^{32}$.

En lo relativo a la segunda razón mencionada, la CPI mantendría plena su capacidad de intervención potencial, habida cuenta de las consecuencias que la aprobación de las leyes de amnistía o punto final han supuesto en la lucha contra la impunidad, uno de los propósitos primordiales explicitados en el Preámbulo del $\mathrm{ER}^{33}$, dado que solo son tres los países iberoamericanos (Venezuela, Brasil y México) que han prohibido expresamente la concesión de amnistías o indultos respecto de algunos ${ }^{34}$, en línea con la sentencia de la Corte Interamericana de Justicia de 14 de marzo de 2001 en el conocido caso Barrios

argentino Jorge Videla o la condena a 6060 años dictada contra varios a altos militares guatemaltecos junto a la imputación al ex dictador Rios Montt del crimen de genocidio, proceso este último aún no resuelto.

${ }^{30}$ Alerta 2012, http://escolapau.uab.es/img/programas/alerta/alerta/13/cap01e.pdf.; Louis Abour, Las guerras de 2013, "Foreing Policy en español", http://www.esglobal.org/index.php?q=las-guerras-de-2013.

${ }^{31}$ Cuadro y mapa de los conflictos actuales en el mundo, www.cidob.org/en/content/ download $/ 24219 / . . . /$ Cuadro+conflictos.p.

${ }^{32}$ El examen de la configuración y tipificación de este delito en la región sobrepasa los propósitos de este trabajo, aunque no nos resistimos a apuntar que Chile, Costa Rica, Guatemala y Nicaragua han tipificado los asesinatos de mujeres cometidos en determinadas circunstancias como femicidio, y El Salvador, México y Perú como feminicidio, expresión esta última utilizada en 2009 por la Corte Interamericana en la sentencia que resolvió el Caso González y otras (Campo Algodonero) vs. México, de 16 de noviembre de 2009. Ana Isabel Garita Vílchez, La regulación del delito de femicidio/ feminicidio en América Latina y el Caribe, edit. Por el Secretariado de la Campaña del Secretario General de las Naciones Unidas ÚNETE para poner fin a la violencia contra las mujeres, Panamá, 2012; Patsilí Toledo Vásquez, Feminicidio, Oficina en México del Alto Comisionado de las Naciones Unidas para los Derechos Humanos, México, 2009.

33 Kai Ambos, Impunidad y Derecho Penal Internacional, Ad hoc, Buenos Aires, 1999; Felipe Gómez Isa, El fenómeno de la impunidad: luces y sombras en América Latina, "Pensamiento Iberoamericano, (In) Seguridad y Violencia en América Latina: un reto para la democracia", n² (2008), pp. 163-185; Miguel Arenas Meza, La contribución de la jurisprudencia de la corte interamericana de derechos humanos a la eliminación de las «leyes de amnistía» en América Latina: un paso decisivo en la lucha contra la impunidad, "Actas XIV Encuentro de Latinoamericanistas Españoles", 2010, pp. 2175-2189.

${ }^{34}$ En el caso de Venezuela la prohibición tiene rango constitucional, pues está contemplada en el artículo 29 de la Constitución. En Brasil y México se limita a determinados crímenes especialmente odiosos, como es el genocidio y en Argentina, pese a no tener rango legal, la jurisprudencia ha afirmado que dichas amnistías son contrarias al Derecho Internacional. Kai Ambos / Ezquiel Malarino, Persecución penal de crímenes internacionales, op. cit., pp. 582 y ss. 
Altos $^{35}$

Con todo, los logros de la CPI en la región no pueden a nuestro juicio ponderarse exclusivamente en atención a su intervención efectiva en casos concretos, puesto que como acabamos de indicar y luego desarrollaremos, a día de hoy ésta se circunscribe al caso colombiano, sino que también debemos tomar en consideración lo que la antigua juez de la CPI Elisabeth Odio Benito ha denominado con gran acierto "logros estructurales"36. Desde esta óptica, la distancia entre lo hecho y lo esperado se acorta sensiblemente ${ }^{37}$, ya que en solo diez años la CPI habría conseguido tres objetivos claves en la región, a saber:

-implantar una ética de respeto a los derechos humanos;

-consagrar la igualdad en la responsabilidad penal internacional por la comisión de crímenes internacionales;

-reforzar la verdad, la justicia y la reparación como elementos esenciales e interrelacionados de la lucha contra la impunidad.

Estos tres logros estructurales se asientan sobre un logro "operacional" no menos relevante, que consiste en el efecto multiplicador positivo que la implementación del ER y sus normas complementarias han provocado en las legislaciones nacionales.

Al apoyar a la CPI, los países iberoamericanos se comprometen con el paradigma de la universalización de la protección de los derechos humanos y reconocen a la Humanidad como sujeto del Derecho Internacional que, en virtud de su capacidad de obrar, puede instar la prevención y sanción de los crímenes más graves de trascendencia para la comunidad internacional en su conjunto $^{38}$.

Simultáneamente, la consagración en el artículo 27 del ER de la improcedencia del cargo oficial, ha abierto la puerta a la posible imputación por la comisión de crímenes internacionales de una amplia categoría de personas físicas (jefes de Estado y de gobierno, parlamentarios, altos funcionarios

${ }^{35}$ Caso Barrios Altos vs. Perú. Sentencia de fondo de 14 de marzo de 2001, p. 41. Esta Corte considera que son inadmisibles las disposiciones de amnistía, las disposiciones de prescripción y el establecimiento de excluyentes de responsabilidad que pretendan impedir la investigación y sanción de los responsables de las violaciones graves de los derechos humanos tales como la tortura, las ejecuciones sumarias, extralegales o arbitrarias y las desapariciones forzadas, todas ellas prohibidas por contravenir derechos inderogables reconocidos por el Derecho Internacional de los Derechos Humanos". http://www.corteidh.or.cr/docs/casos/articulos/seriec 75 esp.pdf.

${ }^{36}$ El futuro de la Corte Penal Internacional. Hacia el X aniversario de la entrada en vigor del Estatuto de Roma, Instituto Internacional Catalán para la Paz, Relatoría del Seminario, Documento 9/2012, p. 16.

${ }^{37}$ Elisabeth Odio Benito, Presentación, "Anuario Iberoamericano de Derecho Internacional Penal, vol. 1", Univ. del Rosario/Tirant lo Blanch/IIH, 2013, pp. 11-28.

${ }^{38}$ Carmen Artigas, El aporte de las Naciones Unidas a la globalización de la ética. Revisión de algunas oportunidades, Unidad Conjunta CEPAL/OACDH (Oficina del Alto Comisionado para los Derechos Humanos) Santiago de Chile, 2001; Xabier Etxeberria, Fundamentación y orientación ética de la protección de los derechos humanos, en La protección Internacional de los derechos humanos en los albores del siglo XXI (Felipe Gómez Isa / José Manuel Pureza (eds.), Universidad de Deusto, 2004, pp. 63-94. 
civiles y militares etc.) que, en virtud de las inmunidades que les confería tanto el derecho internacional consuetudinario como el derecho interno, habrían venido disfrutando de facto de una total impunidad, hasta el extremo de que este fue uno de los impedimentos alegados en México y Chile para retrasar la ratificación del ER, y en El Salvador para justificar su actual posición. Todo ello sin perjuicio de que en la práctica la CPI no haya logrado la colaboración estatal necesaria para ejecutar las dos órdenes de arresto dictadas contra los jefes de Estado en ejercicio de Sudán (Omar al Bashir) y Kenia (Uhuru Kenyatta). No obstante, en lugar de cuestionar o poner en tela de juicio la igualdad en la aplicación de la responsabilidad penal internacional de las personas físicas, la negativa de estas autoridades nacionales no hace sino confirmar que, por una parte, las inmunidades ratione personae quedan desactivadas en los crímenes objeto de la competencia de la CPI, y por la otra, que las normas de derecho interno que establecen estatutos especiales como los aforamientos o regulan las inmunidades personales no son extrapolables al plano internacional ${ }^{39}$.

Por lo que se refiere al logro operacional, no puede ignorarse que la transposición del ER a los ordenamientos internos es una tarea de extrema complejidad y de la larga data, puesto que el mismo reúne en un único instrumento disposiciones que a nivel doméstico están dispersas en normas de diferente rango, desde la constitución a los códigos penales, pasando por las leyes de enjuiciamiento criminal o la normativa relativa al poder judicial. Se calcula que aproximadamente la mitad de los 122 Estados partes en el ER todavía no han adoptado una legislación de desarrollo ${ }^{40}$, la misma ratio que en Iberoamérica, donde el impacto inmediato del ER ha sido relativo, ya que de los 15 países que ratificaron el ER solo la mitad se encuentra en una fase avanzada del proceso de implementación, con resultados ciertamente dispares ${ }^{41}$.

En realidad, no existe un modelo iberoamericano común de transposición, pudiéndose diferenciar en términos comparados tres sistemas distintos, cada uno con ventajas e inconvenientes ${ }^{42}$. El primero consiste en la aprobación de una ley especial omnicomprensiva que prevea todos los aspectos contemplados en

\footnotetext{
39 Aunque el estatuto es taxativo sobre la imposibilidad de invocar inmunidad alguna cuando la CPI ejerce su competencia, ello no significa que derogue las inmunidades personales previstas en el derecho internacional consuetudinario, ni que, de conformidad con el artículo 98 del ER, la CPI pueda forzar a un estado a colaborar con ella si dicha colaboración puede hacer que el estado requerido incurra en responsabilidad internacional por la violación de una obligación vigente respecto a un tercer estado. Isabel Lirola / Magdalena Martín, La Corte Penal Internacional. Justicia versus impunidad, Ariel, Barcelona, 2001, pp. 144 y ss; Carmelo Borrego, La legalidad y los Personales para el enjuiciamiento ante la Corte Penal Internacional, La Corte Penal Internacional: una perspectiva iberoamericana, op. cit., pp. 23 y ss.

${ }^{40}$ Carmen Quesada, Las normas nacionales de adecuación al Estatuto de Roma y de cooperación con la Corte, El futuro de la Corte Penal Internacional, op. cit., p. 31.

${ }^{41}$ Informe de avance del proceso de implementación del Estatuto de Roma, Fundación Konrad Adenauer, mayo de 2011, www.kas.de/rspmexiko.

${ }^{42}$ Lineamientos para la implementación del Estatuto de Roma, Comisión Andina de Juristas, Lima, 2008.
} 
el ER, incluyendo la cooperación ${ }^{43}$, opción inicialmente elegida por tres países: Uruguay, que fue pionero en utilizar este mecanismo (Ley No 18.026 de 11 de octubre de 2006), complementándolo además con otras reformas subsidiarias a efectos de la cooperación con la CPI ${ }^{44}$; Argentina (Ley No. 26.200, de enero de 2007), y Brasil (Lei No. 301 de 2007).

El modelo intermedio opta por una implementación parcial y sistemática del ER, mediante la modificación progresiva de las normas penales y procesales, y ha sido el escogido por Perú (Código Procesal Penal, Decreto Legislativo N 957 de 1 de febrero de 2006); Panamá (Código Penal de mayo de 2007); Ecuador (Ley sobre delitos contra la humanidad de octubre de $2013^{45}$ ), y Colombia, donde el vigente código penal ha incorporado los crímenes objeto de la competencia de la CPI.

El tercer modelo, sin duda el más original, es el seguido en Costa Rica (Ley 8. 271, de mayo de 2002), que ha preferido la aprobación de una ley de remisión que reenvía directamente al ER para su aplicación directa, acompañada de reformas menores en el código penal (ley 8272, de mayo de 2003).

Lamentablemente, en el resto de países iberoamericanos el proceso de implementación del ER es insuficiente, cuando no inexistente. Tal es el caso de Venezuela, primer país en ratificar el ER, pero donde dicho proceso sufre una parálisis total, estando pendiente desde 2005 la aprobación de un nuevo código penal, situación similar a la que se vive en Honduras. En Guatemala y Paraguay ni siquiera se registran proyectos de leyes de implementación. En otros tres países las propuestas existen, pero no acaban de materializarse. Así, en Bolivia la Defensoría del Pueblo presentó en 2006 un proyecto de ley especial de implementación que el Ministerio de Justicia ha utilizado como base para una segunda propuesta aún en trámite. Por su parte, en Chile la situación no es más alentadora, quizás por la tardía ratificación del ER (Ley 20.352, de 30 de mayo de 2009 que reforma la Constitución política de 1980), habiéndose aprobado tan solo una ley previa de 11 de abril de 2009, que tipifica los delitos de genocidio, crímenes de guerra y crímenes de lesa humanidad. Por último,

${ }^{43}$ En este sentido, en el seno de la OEA el Comité Jurídico Interamericano analizó desde 2005 las formas de fortalecer la cooperación con la Corte Penal Internacional, para finalmente, basándose en el Informe CJI/doc.290/08 rev.1 de 3 presentado por el relator especial Herdocia Sacacia, adoptar el 7 de marzo de 2008 una "Guía de principios generales y pautas en materia de cooperación de los Estados con la Corte Penal Internacional”, con directrices generales para que los miembros de la OEA implementaran sus normas a la luz de las particularidades propias de los ordenamientos internos, http://www.oas.org/es/sla/ddi/docs/CJI doc_293-08 rev1 esp.pdf.

44 Pablo Galain Palermo, The Prosecution of International Crimes in Uruguay, "International Criminal Law Review”, no 4 (2010), pp. 601-618.

${ }^{45}$ A resultas de dicha ley y de los resultados de la Comisión de la Verdad creada por el presidente Correa para investigar los crímenes internacionales cometidos entre 1984 y 1988, en octubre de 2013 la juez Blazio aceptó la petición de la Fiscalía y ordenó el arresto de seis generales y tres coroneles retirados por crímenes de tortura, violencia sexual y desaparición forzadas de tres guerrilleros en 1985. 
en México el Senado rechazó en diciembre de 2009 el proyecto de Ley de Cooperación con la Corte Penal Internacional, pese a lo cual el gobierno se comprometió durante la Conferencia de Kampala a presentar una propuesta legislativa de enmienda del código penal que sigue en el aire.

En cualquier caso, sin perjuicio de que la influencia del ER en los ordenamientos internos de la región, y las reformas legislativas de naturaleza sustantiva y procesal acometidas distan de ser las esperadas ${ }^{46}$, es innegable que ha generado ya un primer impacto positivo, al consagrar el deber estatal de investigar y sancionar las violaciones graves a los derechos humanos y del derecho internacional humanitario. Los países iberoamericanos han adquirido así un "compromiso diferenciado" de persecución de los crímenes internacionales ${ }^{47}$, que se traduce en la asunción de la llamada complementariedad positiva, de manera que en caso de que incumplan dicho deber por falta de voluntad o de capacidad, son conscientes de que la CPI se encargará de llevar a cabo los procesamientos. En otras palabras, las jurisdicciones nacionales asumen la responsabilidad primordial y primaria en el enjuiciamiento de los crímenes internacionales, lo que en Iberoamérica implica el fortalecimiento de los sistemas de justicia nacionales, incluyendo la aceptación del principio de jurisdicción universal ${ }^{48}$, así como la reactivación de los mecanismos regionales de protección de los derechos humanos. En particular, este compromiso diferenciado comporta la fertilización cruzada o influencia recíproca entre la labor de la Corte Interamericana de Derechos Humanos y la $\mathrm{CPI}^{49}$, sobre todo en relación con la protección de las víctimas.

Por consiguiente, el efecto inmediato del ER en la región estriba no tanto en forzar a los países iberoamericanos a modificar su derecho interno para cumplir con su deber de implementación, y que sean sus propios tribunales los

${ }^{46}$ En particular todavía se registran importantes lagunas en los derechos internos en lo relativo a la implementación de los mecanismos de cooperación con la CPI, y en la tipificación de los delitos relacionados con la administración de justicia y, sobre todo, de los crímenes de naturaleza sexual. Corte Penal Internacional. Desafios en materia de justicia de género en la región, Proyecto Regional CPI y Justicia de Género, Santiago de Chile, 2003; Magdalena Martin / Isabel Lirola, Los crímenes de naturaleza sexual en el Derecho Internacional Humanitario, Informe 8/2013, http://www20.gencat. cat/portal/site/icip/.

${ }^{47}$ Expresión utilizada por Ezequiel Malarino, Persecución penal, op. cit., Informe sobre Argentina.

${ }^{48}$ José R. Grijalva Eternod, Las violaciones a los derechos humanos y el principio de jurisdicción universal, en Joaquín Mejía / Víctor Fernández (Coord.), El golpe de Estado en Honduras desde una perspectiva de los derechos humanos, Editorial Casa San Ignacio/MADJ, Tegucigalpa, 2010. La incorporación del principio de jurisdicción universal en las legislaciones de los países iberoamericanos ha traído consigo la apertura de casos impensables en el pasado, como la extradición de Fujimori por parte de Chile, la de Cavallo por parte de México, o más recientemente la solicitud de extradición formulada en diciembre de 2013 por la juez argentina Maria Servini a España contra policías y guardias civiles franquistas acusados de crímenes de tortura. Manuel Ollé, Contribución de la Justicia Universal a Latinoamérica, "Revista Electrónica Iberoamericana", vol. 4, n² (2010). Samantha Salsench i Linares, Francoism Facing Justice. Enforced Disappearances before Spanish Courts, "Journal of International Criminal Justice" no 11 (2013), pp. 463-483.

${ }^{49}$ Grupo Latinoamericano de Estudios sobre Derecho Penal Internacional, Sistema Interamericano de protección de los derecho humanos y derecho penal internacional, Fundación Konrad Adenauer, Uruguay, 2011. 
que enjuicien los crímenes internacionales cometidos en su territorio, como en el descrito por Ezequiel Malarino: "el incentivo de no perder la soberanía, esto es, el incentivo que significa para todo Estado poder juzgar por sí mismo sus conflictos internos (...) La posible activación de la competencia de la CPI (y la consiguiente pérdida de la porción de soberanía que significa la jurisdicción) opera, por tanto, como un estímulo para la implementación estatal"50. A su vez para la CPI, y en concreto para la Fiscalía, el desarrollo de la complementariedad positiva es una estrategia de acción crucial, habida cuenta de lo limitado de sus recursos materiales y humanos, y de la dificultad para investigar todas las situaciones sin caer en juicios fantasmas.

Ese es justo el reto pendiente, el desafío actual en Iberoamérica: la concreción y materialización de la complementariedad positiva ${ }^{51} \mathrm{y}$ el reconocimiento de que, conforme al artículo 17.1, corresponde a la CPI la valoración continuada de la falta de voluntad o capacidad de un país iberoamericano en concreto para llevar a cabo las investigaciones y los enjuiciamientos de los crímenes internacionales objeto de su competencia.

\section{Los exámenes preliminares de la Fiscalía en Colombia ${ }^{52}$ y Honduras $^{53}$, que}

${ }^{50}$ Ezequiel Malarino, Derechos Humanos y Derecho Penal. Estudio sobre el sistema interamericano de derechos humanos y el derecho penal internacional, Colección Internacional $\mathrm{n}^{\circ} 39$, Pontificia Universidad Javeriana/ Grupo Editorial Ibáñez, Bogotá, 2012, p. 304.

${ }^{51}$ En consonancia con la RC/Res.1, Complementariedad, adoptada en la Conferencia de Revisión de Kampala, http://www.icc-cpi.int/iccdocs/asp_docs/ASP9/OR/RC-11-Part.I-SPA.pdf.

${ }^{52}$ Sobre la actuación en Colombia. Oficina del Fiscal. Situación en Colombia, Reporte Intermedio, Noviembre 2012. La bibliografía sobre el caso colombiano es ya muy extensa. Destacamos, sin ánimo de exhaustividad: Pablo Rueda Saiz, Algunas reflexiones sobre la corte penal internacional en relación con el proceso de paz colombiano, "Revista Colombia Internacional", n43 (1998), p. 41-59; Bárbara Direito, Latinoamérica y la CPI: afrontar la impunidad en Colombia, "Araucaria. Revista Iberoamericana de Filosofía, Política y Humanidades", nº 20 (2008), págs. 137-154; Héctor Olásolo, Instituto Iberoamericano de La Haya para la Paz, los Derechos Humanos y la Justicia Internacional IHH, Derecho y Humanidades, nº 18 (2011), pp. 15-21; Héctor Olásolo, El principio de complementariedad y las estrategias de actuación de la corte penal internacional en la fase de examen preliminar: ¿por qué la corte penal internacional mantiene su examen preliminar, pero no abre una investigación, sobre la situación en Colombia?, "Revista Electrónica de Estudios Internacionales," $n^{\circ} 24$ (2012), http://www.reei.org/index.php/revista/num24/articulos/principio-complementariedadestrategias-actuacion-corte-penal-internacional-fase-examen-preliminar-corte-penal-internacionalmantiene-su-examen-preliminar-pero-abre-una-investigacion-sobre-situacion-colombia.

${ }^{53}$ Respecto a Honduras, la CPI ha manifestado expresamente en menos de dos años su decisión de continuar con el examen preliminar en curso. La primera ocasión en diciembre de 2012, Actuaciones de la Fiscalia en Honduras. The Office of the Prosecutor Report on Preliminary Examination Activities, 13 December 2012; p. 14. "Conclusion and Next Steps: 63, The Office will continue to evaluate, including on the basis of any additional information received, whether the alleged crimes committed in Honduras between June 2009 and January 2010 amount to crimes against humanity, so as to enable a final determination in due course"; La segunda ocasion tuvo lugar a mediados de 2013: Informe de la Corte Penal Internacional correspondiente a 2012713, A/68/314, 13 de agosto de 2013, p. 17. Honduras, páragrafo 93: "Durante el período que se examina, la Fiscalía siguió colaborando con organizaciones no gubernamentales, interesados regionales y remitentes de comunicaciones en virtud del artículo 15 a fin de recabar información complementaria sobre la situación en Honduras. La Fiscalía actualizó su análisis, entre otras cosas, sobre la base del informe de la Comisión de Verdad alternativa publicado el 3 de octubre de 2012. La Fiscalía siguió evaluando, entre otras cosas en función de la información complementaria recibida, si los presuntos delitos cometidos en Honduras desde junio de 
no vamos a examinar aquí, además de mostrar que se trata de un reto real y no retórico, han sacado a la luz la doble cara de la complementariedad positiva. Por una parte, su función activadora e impulsora de la justicia nacional. Por la otra, el temor de que sea usada torticeramente para menoscabar o neutralizar la competencia de la CPI, perpetuando la impunidad. Al margen de la complejidad de los hechos y los problemas jurídicos previos que rodean a ambos casos, en nuestra opinión hay que partir de la siguiente premisa: al afrontar el dilema entre justicia y paz ${ }^{54}$, conviene recordar que la CPI es un órgano judicial y debe optar siempre por la primera, por lo que si los tribunales nacionales no pueden o no quieren, la CPI debe encontrar el "momento adecuado" para juzgar, y si procede condenar, a los presuntos autores de crímenes internacionales. En otras palabras, a la CPI compete asegurarse de que en Iberoamérica no exista ninguna brecha en la lucha contra la impunidad ${ }^{55}$.

\section{A modo de reflexión final}

De todo lo hasta aquí expuesto se desprende que Iberoamérica y la CPI mantienen desde hace más de dos décadas una interacción compleja y viva, con luces y sombras.

A efectos de clarificación y resumen, en la aportación de Iberoamérica a la CPI pueden distinguirse dos etapas.

En una primera fase, el compromiso de la región con la CPI fue inequívoco, tanto en el plano estatal individual como en el multilateral, canalizado a través de la acción de la OEA. Los países iberoamericanos apoyaron de manera activa el proceso de establecimiento de la CPI, desde la negociación a la ratificación, dejando su impronta en diversos aspectos, sustantivos, procesales e incluso personales del ER.

Esta trayectoria positiva se mantuvo hasta la Conferencia de Kampala, que marca el inicio de una segunda fase en la que se produce un punto de inflexión. Pese a que se atenúan los principales obstáculos endógenos (tránsito de regímenes dictatoriales a democracias incipientes), y exógenos (disminución de la hostilidad contra la CPI ejercida por los Estados Unidos de América), en contra de lo esperado empiezan a aparecer signos de crisis en la unidad de

2009 constituían crímenes de lesa humanidad". Joaquín A. Mejía R, Una Mirada Interamericana a la Justicia Penal Internacional: El Caso de Honduras, La Corte Penal Internacional: Una perspectiva Latinoamericana, op. cit., pp. 105 y ss.

${ }^{54}$ Christian Wolffhügel Gutiérrez, El principio de complementariedad y la ley de justicia y paz: principales desafios. ¿La Corte Penal Internacional podrá ejercer su facultad de intervención complementaria?, Colección Estudios de Derecho Penal, Universidad Sergio Arboleda, Bogotá, 2011.

${ }_{55}$ Balance de la justicia penal internacional. Balance sobre el principio de complementariedad: salvar la brecha de la impunidad a RC/ST/CM/1.(ICC -ASP/8/20/Add.1), parte II ICC ASP/8/Res.9, anexo IV, http://www.icc-cpi.int/iccdocs/asp_docs/RC2010/RC-11-Annex.V.c-SPA.pdf. 
acción iberoamericana. El apoyo incondicional se debilita a medida que la CPI amplía sus miras más allá de los confines del continente africano, sobre todo a raíz de los exámenes preliminares abiertos por la Fiscalía sobre la situación en Colombia y en Honduras, prueba inequívoca de que la CPI tiene la firme voluntad de ejercer, conforme a su jurisdicción complementaria, un papel de garante último de la lucha contra la impunidad en la región.

Al tratarse de una interacción recíproca, la aportación de Iberoamérica a la CPI concurre con la contribución que la implementación del ER supone para los países de la región. Tal y como ha quedado de manifiesto, además de favorecer el arraigo definitivo de la ética de respeto a los derechos humanos, tan necesaria habida cuenta del legado perverso de las dictaduras y los conflictos armados, la puesta en marcha de la CPI tiene un efecto útil en la transformación de los sistemas de justicia y los ordenamientos jurídicos internos y en su adecuación definitiva al Derecho Internacional. Ciertamente este efecto está siendo desigual y no puede darse por finalizado, en la medida en que en buena parte de la región sigue siendo necesaria la adopción de nuevas normas y/o la reforma de las ya existentes.

Pese a ello, los avances derivados de la incorporación e implementación del ER, sumados a la derogación de las amnistías, han multiplicado la capacidad de los tribunales iberoamericanos para enjuiciar los crímenes internacionales, incluyendo aquellos olvidados como los de naturaleza sexual, si bien persisten obstáculos clásicos como los derivados de la debilidad de los sistemas de justicia o las dificultades probatorias, junto a otros nuevos, como la reducción sistemática de las penas o los indultos a los condenados por la perpetración de dichos crímenes ${ }^{56}$. En este escenario, la CPI, a la par que aprueba y apoya los procesos transicionales encaminados a la consolidación de la paz y la democracia, como demuestran las declaraciones tras las sucesivas visitas oficiales que la CPI cursó a este país en 2013, no renuncia a ejercer el mandato en la prevención y castigo de los crímenes más graves de trascendencia para la comunidad internacional en su conjunto, mandato que los países iberoamericanos le han encomendado.

${ }^{56}$ Digesto de Jurisprudencia Latinoamericana sobre crimenes de Derecho Internacional, vol. II, Fundación para el debido proceso, Washington, 2013. 


\section{Bibliografía}

Abour Louis, Las guerras de 2013, "Foreing Policy en español”, 2 enero 2013. Ambos Kai, Impunidad y Derecho Penal Internacional, Ed. Ad hoc, Buenos Aires, 1999.

-¿Corte Penal Internacional o Corte Africana?, "Diario ABC," 28 de octubre de 2013.

Arieff Alexis, International Criminal Court Cases in Africa: Status and Policy Issues. Congressional Research Service, (2009), pág. 22 y ss.

Arenas Meza Miguel, La contribución de la jurisprudencia de la corte interamericana de derechos humanos a la eliminación de las «leyes de amnistía» en América Latina: un paso decisivo en la lucha contra la impunidad, "Actas XIV Encuentro de Latinoamericanistas Españoles", 2010, pág. 2175-2189.

Artigas Carmen, El aporte de las Naciones Unidas a la globalización de la ética. Revisión de algunas oportunidades, Unidad Conjunta CEPAL/ OACDH (Oficina del Alto Comisionado para los Derechos Humanos), Santiago de Chile, 2001.

Benvenuto Francesca, Revuelta de los países africanos: El Tribunal Penal Internacional acusado, "Le Monde Diplomatique en español", no 217 , octubre (2013).

Boeglin Nicolás / Julia Hoffmann / Juan C. Sáinz-Burgo (ed.), La Corte Penal Internacional: una perspectiva latinoamericana, CPI-Universidad para la paz, Costa Rica, 2012.

- A 10 años de la entrada en vigor del Estatuto de Roma: Breves reflexiones desde una perspectiva latinoamericana, "Revista Judicial de Costa Rica", n 107 (2013), págs. 13-21.

Borrego Carmelo, La legalidad y los Personales para el enjuiciamiento ante la Corte Penal Internacional, La Corte Penal Internacional: una perspectiva iberoamericana, op. cit., pág. 23 y ss.

Cárdenas Claudia / Fernández Karinna, La Corte Penal Internacional y sus primeros 10 años: un enfoque práctico, Thomson Reuters, 2013.

Corte Penal Internacional. Desafíos en materia de justicia de género en la región, Proyecto Regional CPI y Justicia de Género, Santiago de Chile, 2003.

Delmas-Marty Mireille, Ambiguities and Lacunae.The International Criminal Law ten years on, "Journal of International Criminal Justice", 11 (2013), pág.553-561.

Derecho Internacional Humanitario y Derechos Humanos. Reflexiones sobre el conflicto colombiano, Castor Díaz / Carlos R. Fernández Liesa / José L. Rodríguez (dir.), Civitas-Thomson Reuters, 2013. 
Digesto de Jurisprudencia Latinoamericana sobre crímenes de Derecho Internacional, vol. II, Fundación para el debido proceso, Washington, 2013.

Direito Bárbara, Latinoamérica y la CPI: afrontar la impunidad en Colombia, "Araucaria. Revista Iberoamericana de Filosofía, Política y Humanidades", no 20 (2008), págs. 137-154.

El futuro de la Corte Penal Internacional. Hacia el X aniversario de la entrada en vigor del Estatuto de Roma, Instituto Internacional Catalán para la Paz, Relatoría del Seminario, Document 9/2012.

Etxeberria Xabier, Fundamentación y orientación ética de la protección de los derechos humanos, en La protección Internacional de los derechos humanos en los albores del siglo XXI (Felipe Gómez Isa / José Manuel Pureza (eds.), Universidad de Deusto, 2004.

Federación Internacional de Derechos Humanos, Corte Penal Internacional 2002-2012: 10 años, 10 recomendaciones para una Corte Penal Internacional eficaz e independiente.

Fernández Arribas Gloria, Entrada en vigor y ejercicio por la Corte Penal Internacional de la jurisdicción sobre el crimen de agresión, "Revista Electrónica de Estudios Internacionales" no 25 (2013).

Fernández de Gurmendi Silvia, El aporte de Iberoamérica a la CPI, en Héctor Olásolo/ Salvador Cuenca, Perspectiva Iberoamericana sobre la Justicia Penal Internacional, Tirant Lo Blanch, Valencia, 2012, pág. 15-18.

Galain Palermo Pablo, The Prosecution of International Crimes in Uruguay, "International Criminal Law Review”, no 4 (2010), págs. 601 - 618.

García Ramírez Sergio, Cuestiones constitucionales a propósito de la Corte Penal Internacional, "Cuestiones Constitucionales", nº 6 (2002), pág. 175-189.

Garita Vilchez Ana Isabel, La regulación del delito de femicidio/feminicidio en América Latina y el Caribe, edit. Por el Secretariado de la Campaña del Secretario General de las Naciones Unidas ÚNETE para poner fin a la violencia contra las mujeres, Panamá, 2012.

Guerrero Silva Belissa, El Estatuto de Roma y su constitucionalidad en el Salvador, "Cuadernos Electrónicos Derechos Humanos y Democracia", no 4 (2008), pág. 33-56.

Gómez Isa Felipe, El fenómeno de la impunidad: luces y sombras en América Latina, "Pensamiento Iberoamericano, (In) Seguridad y Violencia en América Latina: un reto para la democracia", nº 2 (2008), pág. 163-185.

Guevara José A. / Dalmaso Narciso, La Corte Penal Internacional. Una visión iberoamericana, Porrúa-Universidad Iberoamericana, México, 2005. 
Goodman Rachel / Mncwabe Nokukhanya, International Criminal Justice in Africa: Neocolonial agenda or strengthened accountability?, "African Transitional Justice Research Network”, October (2011).

González Gálvez Sergio, México y la Corte Penal Internacional, "Revista Mexicana de Política Exterior”, no 55-56 (1999), págs.44-67.

Grijalva Eternod José R, Las violaciones a los derechos humanos y el principio de jurisdicción universal, en Joaquín Mejía/ Víctor Fernández (Coord.), El golpe de Estado en Honduras desde una perspectiva de los derechos humanos, Editorial Casa San Ignacio/MADJ, Tegucigalpa, 2010.

Iberoamérica y la Corte Penal Internacional: debates, reflexiones y preguntas, Luis Martí Mingarro/ Fernando Carrillo Flórez (ed.), Colección textos de jurisprudencia, Bogotá, 2006.

Informe de avance del proceso de implementación del Estatuto de Roma, Fundación Konrad Adenauer, mayo de 2011.

Isanga Joseph, The International Criminal Court ten years later: appraisal and perspective, "Cardozo Journal of International and Comparative Law", 21 (2013), pág. 235-323.

Lineamientos para la implementación del Estatuto de Roma, Comisión Andina de Juristas, Lima, 2008.

Lirola Isabel/ Martín Magdalena, La Corte Penal Internacional. Justicia versus impunidad, Ariel, Barcelona, 2001.

Malarino Ezequiel, Derechos Humanos y Derecho Penal. Estudio sobre el sistema interamericano de derechos humanos y el derecho penal internacional, Colección Internacional $\mathrm{n}^{\circ} 39$, Pontificia Universidad Javeriana/ Grupo Editorial Ibañez, Bogotá, 2012, pág 304.

Martin Magdalena/ Lirola Isabel, Los crímenes de naturaleza sexual en el Derecho Internacional Humanitario, Informe 8/2013, http://www20. gencat.cat/portal/site/icip/.

Martínez Guerra Amparo Corte Penal Internacional: balance de una década, "Política Exterior" nº 148, julio/agosto (2012), pág.. 22-27.

Minoves-Triquell Juli, ONG y pequeños estados en el establecimiento y consolidación de la CPI, "Revista CIDOB d'AfersInternacionals", n 101 (2013) pág. 177-193.

Mejía Joaquín, Una Mirada Interamericana a la Justicia Penal Internacional: El Caso de Honduras, La Corte Penal Internacional: Una perspectiva Latinoamericana op. cit., p. 105 y ss.

Odio BenitoElisabeth, Presentación, Anuario Iberoamericano de Derecho Internacional Penal, vol. 1., Univ. del rosario/Tirant lo Blanch/IIH, 2013

Olásolo Héctor, Instituto Iberoamericano de La Haya para la Paz, los Derechos Humanos y la Justicia Internacional IHH, Derecho y Humanidades, $\mathrm{n}^{\circ} 18$ (2011), pp. 15-21; 
-El principio de complementariedad y las estrategias de actuación de la corte penal internacional en la fase de examen preliminar: ¿por qué la corte penal internacional mantiene su examen preliminar, pero no abre una investigación, sobre la situación en Colombia?, "Revista Electrónica de Estudios Internacionales", no 24 (2012).

Ollé, Manuel, Contribución de la Justicia Universal a Latinoamérica, "Revista Electrónica Iberoamericana", vol. 4, n² (2010).

Quesada, Carmen (coord.), Décimo Aniversario de la Corte Penal Internacional, "Tiempo de Paz", (2012).

-Las normas nacionales de adecuación al Estatuto de Roma y de cooperación con la Corte, El futuro de la Corte Penal Internacional, op. cit., pág. 31

Rueda Sáiz Pablo, Algunas reflexiones sobre la corte penal internacional en relación con el proceso de paz colombiano, "Revista Colombia Internacional", nº43 (1998), pág. 41-59.

Salsench i Linares Samantha, FrancoismFacing Justice. Enforced Disappearances before Spanish Courts, "Journal of International Criminal Justice" no 11 (2013), págs. 463-483.

Scheffer David, America's Embrace of the International Criminal Court, JURIST - Forum, July 2, 2012.

Sistema Interamericano de protección de los derecho humanos y derecho penal internacional, Fundación Konrad Adenauer, Uruguay, 2011.

Toledo Vásquez Patsilí, Feminicidio, Oficina en México del Alto Comisionado de las Naciones Unidas para los Derechos Humanos, México, 2009.

Vaquerazo Nelson / Martínez Jaime, El Salvador: Informes Nacionales, en Kai Ambos y Ezequiel Malarino(ed), Persecución Penal Nacional de Crímenes Internacionales en América Latina y España, Fundación Konrad Adenauer, Uruguay, 2003, pág. 305-335

Varda Francesca, Working together for justice, La Corte Penal Internacional: una perspectiva Latinoamericana, op. cit., pp. 384-403.

Wolffhügel Gutiérrez, Christian, El principio de complementariedad y la ley de justicia y paz: principales desafios. ¿La Corte Penal Internacional podrá ejercer su facultad de intervención complementaria?, Colección Estudios de Derecho Penal, Universidad Sergio Arboleda, Bogotá, 2011. 
